\title{
44559 - ANESTHETIC MANAGEMENT OF A PATIENT UNDERGOING LAPAROSCOPIC ADRENALECTOMY FOR DOPAMINE SECRETING PHEOCHROMOCYTOMA
}

\section{Andrea Dower, Queen Elizabeth II Health Sciences Centre, Halifax, NS, Canada;}

PURPOSE: Pheochromocytoma is a well-recognized entity within the practice of anesthesiology with particular considerations. Pheochromocytomas that secrete only dopamine (DA) are rare. This diagnosis is also complicated as such tumors may not produce a positive metaiodobenzylguanidine (MIBG) scan as seen in classical pheochromocytomas $(1,2)$. Previous case reports have suggested that alpha blockade in such patients may be unnecessary and in fact harmful(1,3). We present a rare case report of a patient with a DA-secreting pheochromocytoma, who underwent laparoscopic resection without the use of alpha blockade. CLINICAL FEATURES: Written informed consent for chart review and case report submission were granted by the patient as per institutional guidelines. A 40 year old $77 \mathrm{~kg}$ Asian male was assessed in the pre-admission clinic two days prior to laparoscopic adrenalectomy. This patient had an asymptomatic $5.3 \mathrm{~cm}$ right adrenal mass that had been discovered incidentally 11 months prior to presentation. The patient had not experienced any headaches, palpitations, diaphoresis, tremor, or hypertension. Pre-operative investigations included a $24 \mathrm{hr}$ urine collection, which revealed normal epinephrine and norepinephrine, but elevated DA at 3160nmol (normal $<2612 \mathrm{nmol}$ ). Pre-operative MIBG scan was negative. Pre-operative alpha blockade had not been initiated, and was not instituted in the pre-operative clinic as the patient was normotensive and euvolemic.

On the day of surgery, large bore IV access was established. He was pre-oxygenated and general anesthesia was induced using midazolam, sufentanil, propofol, and rocuronium intravenously. Neuromuscular blockade was confirmed and the patient remained hemodynamically stable despite the need for two laryngoscopy attempts. A left radial arterial line was established following induction.

Anesthetic maintenance consisted of nitrous oxide, desflurane and a remifentinal infusion. Intraoperative hemodynamics remained stable and no alpha-blockade or vasopressor support were required. He received a total of 3.5L of crystalloid and was extubated uneventfully at the conclusion of the case. He was monitored routinely in the PACU and subsequently discharged to the ward. The patient completed an uneventful recovery and was discharged home. CONCLUSION: Pheochromocytomas secreting only DA are a rare but recognized clinical entity. Traditional anesthetic management of patients with catecholamine secreting tumors suggests that patients be adequately alpha-blocked prior to commencement of surgery. However, in pheochromocytomas secreting only DA, this may be unnecessary and perhaps deleterious. We present a rare case report of a patient with a DA-secreting pheochromocytoma, who underwent laparoscopic resection without the use of alpha blockade and had an uneventful perioperative course.

REFERENCES: 1) Surgery 1986 100(6): 1154-1162 2) World J. Surg. 2005 29: 909-913 3) South Med J 2003 96(9): 914-917 